\title{
Stationary Myoelectric Spectra from a Nonparametric, Low Bias, and Low Variance Estimator
}

\author{
Kristin A. Farry, NASA/Johnson Space Center/ER, Houston, Texas 77058 USA \\ Richard G. Baraniuk and Ian D. Walker, Electrical and Computer Engineering, Rice University, \\ Houston, Texas 77251-1892 USA
}

Abstract. We explain Thomson's crude multiple window method (MWM), a nonparametric spectral estimator with low bias and variance. We then compare its output to Hamming-windowed periodogram and autoregressive (AR) method outputs for a sample myoelectric signal. Our goal is a spectral estimator to embed in a myoelectric controller.

Infroduction. Myoelectric signal spectrum estimation requires a method that (1) works well on small (and hence relatively stationary) windows of a single realization of the signal; (2) does not suffer from bias and variance that obscures the spectral features we are trying to detect; and (3) incorporates no incorrect assumptions about the structure of the signal. The periodogram suffers from substantial bias and variance [1]. Autoregressive (AR) model-based estimators [1], [2] have lower variance than the periodogram, but their all-pole assumption matches certain motion artifacts better than the underlying physiological signal. Thus, motion-specific detail can be lost as the model converges on motion artifact. Here, we test a more recently developed nonparametric spectral estimator, Thomson's multiple window method (MWM) [3], which gives lower bias and variance than the periodogram without introducing a potentially misleading model [3], [4].

Methods. Instead of one ad hoc window such as the Hamming window as is often used in the periodogram, we apply a set of $N$-point windows to the data that are (1) mutually orthogonal and (2) optimally concentrated in frequency. The windows -- discrete prolate spheroidal sequences (DPSSs) -- are the inverse discrete time Fourier transforms of the eigenfunctions of a cascaded band-limiting (to $[-W, W]$ ) and time-limiting (to $[0, N]$ ) operation [5]. The crudest MWM power spectral density estimate on a length- $N$ data window is

$$
\hat{P}_{x x}(f)=\frac{1}{2 N W} \sum_{k=0}^{2 N W-1} \frac{1}{\lambda_{k}}\left|x_{k}(f)\right|^{2},
$$

where $x_{k}(f)$ are the eigenspectra

$$
\left|x_{k}(f)\right|^{2}=\left|\sum_{n=0}^{N-1} x[n] v_{k}[n] e^{-j 2 \pi f n}\right|^{2} .
$$

$\nu_{k}[n]$ is the $k$ th DPSS window and $\lambda_{k}$ is its corresponding eigenvalue. $W$ is the resolution of the estimate. We use only the largest $K=2 N W$ eigenvalues and their corresponding eigenfunctions in the estimate; $K$ is the tradeoff between the bias and additional spectral information contributed by the higher order DPSSs. Larger $K$ gives smaller variance but larger $W$ and bias. Figure 1 shows the $K=4$ case computations.

Results and Discussion. Figure 2 compares the Hamming-windowed periodogram, the AR (model order set at 5 by Akaike Information Criterion [1]), and the crude Thomson's estimates for a surface-measured Abductor Pollicis Longus and Extensor Pollicis Brevis monopolar myoelectric signal ( $6 \mathrm{~mm}$ recessed wet electrode, bandpass filtered 3-300 Hz, sampled at $1000 \mathrm{~Hz}$ ). The sample contains some motion artifact, as we measured it during thumb abduction.

Conclusions. Thomson's MWM estimates more closely match what we expect from the physiological processes producing the myoelectric signal [6] than the periodogram and AR estimates. Crude MWM estimates have significantly lower bias and variance than the periodogram, and lower bias than the AR estimate in the presence of noise or poor model fit. Averages over many trials of the same motion contrast the periodogram-MWM variance (Figure 3 ).

Thomson developed a lower bias version of this spectral estimation approach, the adaptive MWM, as well as tests for embedded harmonics that, in the myoelectric analysis application, give insights into motion artifact [4].

Acknowledgements. Support included a NSF Graduate Fellowship, a Zonta International Fellowship, and two NSF Grants (MSS9024391 and MIP9457438). NASA/JSC also provided funding (NAG9-740) and research equipment.

\section{References}

1. S. M. Kay. Modern Spectral Estimation, (Englewood Cliffs, New Jersey: Prentice-Hall, Inc., 1988).

2. D. Graupe et al. "A Microprocessor System for Multifunctional Control of Upper-Limb Prostheses via Myoelectric Signal Identification," IEEE Trans. on Auto. Control, AC-23, No. 4, pp. 538-544.

3. D. Thomson. "Spectrum Estimation and Harmonic Analysis," Proc. of the IEEE, Vol. 70, No. 9, pp. 1055-1096. 4. K. Farry. Issues in Myoelectric Teleoperation of Complex Artificial Hands, Ph.D Dis., Elec. and Computer Eng., Rice University, Nov 1994.

5. A. Drosopoulis \& S. Haykin. Adaptive Radar Estimation, Haykin and Steinhardt, ed. (John Wiley \& Sons, Inc., 1992). 6. J. Basmajian \& C. DeLuca. Muscles Alive, (Baltimore: Williams and Wilkins, 1985), 5th Ed. 
Figure 1: Thomson's multiple window method, crude version, for $N W=2$.

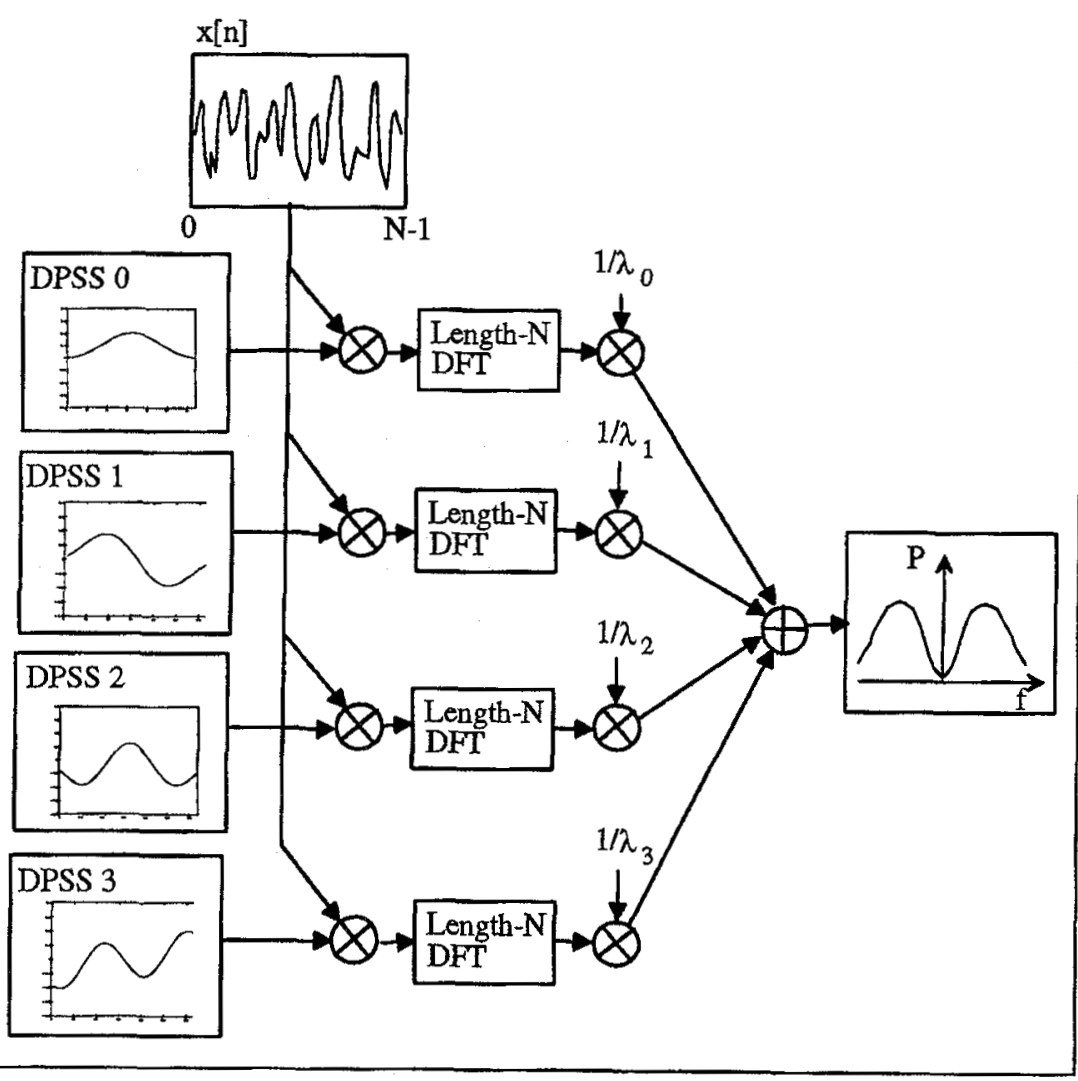

Figure 3: Thumb abduction myoelectric Hamming periodogram (dashed line) and crude MWM (solid line) normalized spectra standard deviation comparison (60 cases, $64 \mathrm{~ms}$ window).

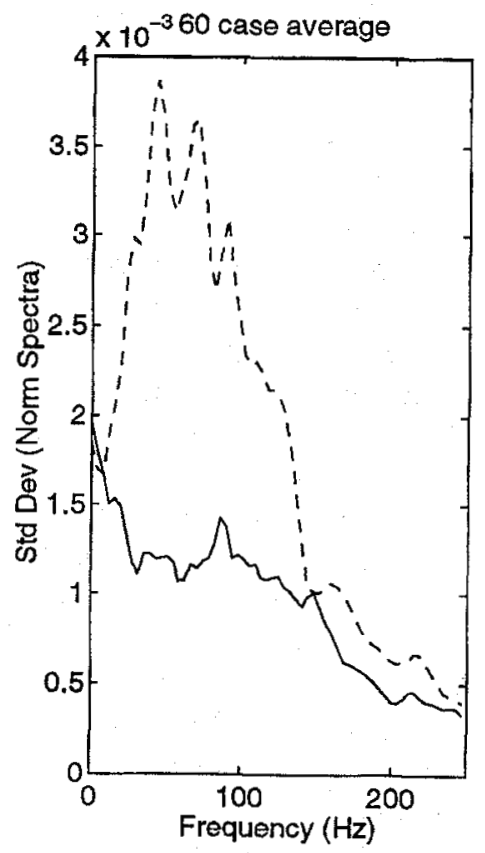

Figure 2: (a) $64 \mathrm{~ms}$ of a sample thumb abduction myoelectric signal and (b) its Hamming periodogram (dotted line), autoregressive with model order $=5$ (dashed line), and Thomson 's crude MWM (solid line) spectral estimates.
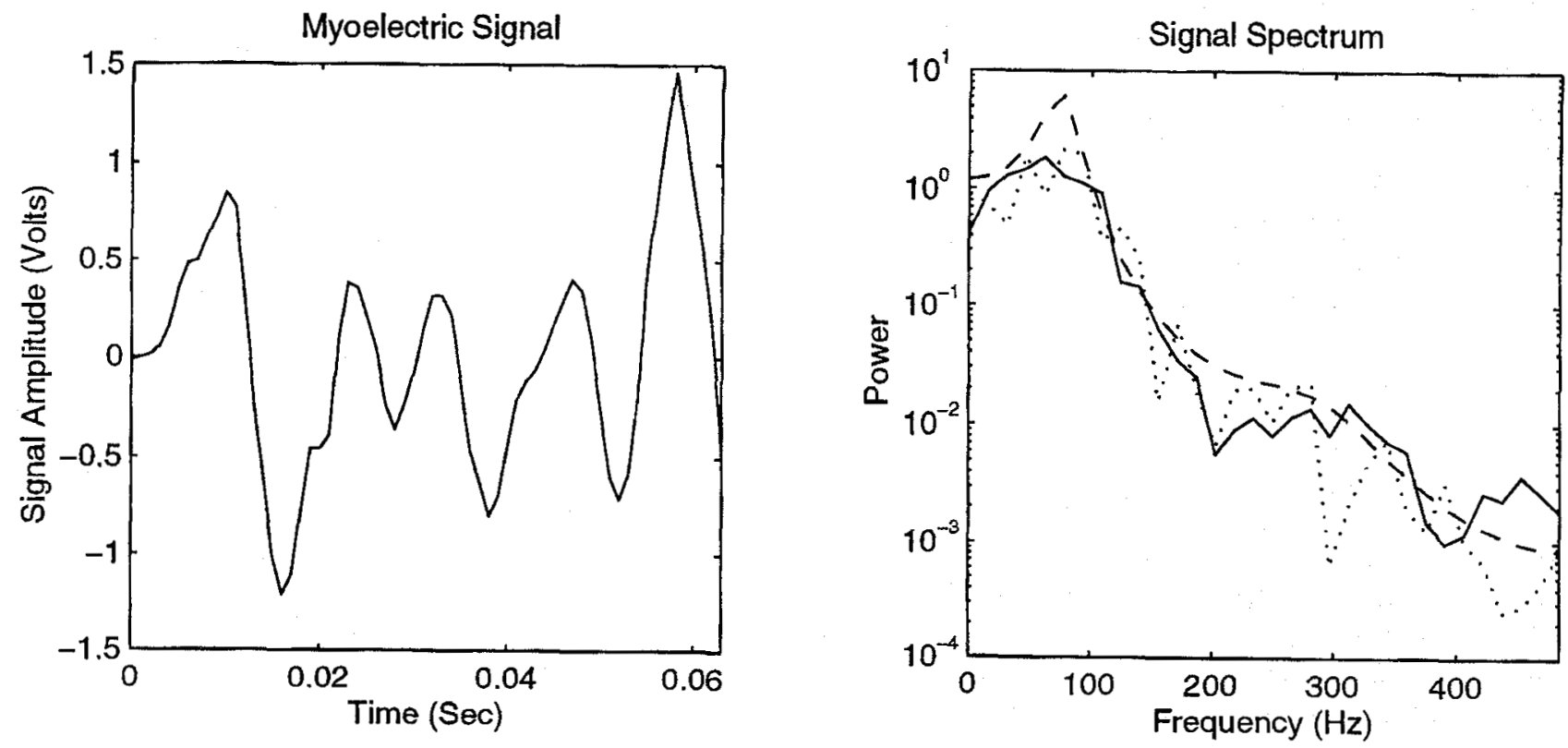